\title{
Industrie des corps gras et changement climatique
}

Fabrice BOSQUE

Claire VERNIOLE

Xavier PAGES

ITERG, Département Technologie

\& Environnement, 11 rue Gaspard Monge -

Parc Industriel Bersol 2, 33600 Pessac - France

$<$ f.bosque@iterg.com>

\section{L'effet de serre et ses conséquences}

La température moyenne de la planète résulte de l'équilibre entre deux flux : le rayonnement qui lui parvient du soleil et le rayonnement absorbé par le sol et l'atmosphère, réémis sous forme de rayonnement infrarouge vers l'espace, contribuant ainsi à en réchauffer les couches inférieures. Agissant telles les vitres d'une serre, certains gaz, appelés gaz à effet de serre (GES), présents naturellement en faibles quantités dans l'atmosphère, interfèrent avec les rayons de l'infrarouge, les empêchant de s'échapper vers l'espace, ce qui provoque une hausse des températures. La répartition de la chaleur au niveau du sol dépend de la quantité de GES présents dans I'atmosphère. Sans eux, la température moyenne serait de $-18{ }^{\circ} \mathrm{C}$ et la Terre serait invivable. Leur présence amène cette température à $15^{\circ} \mathrm{C}$. L'effet de serre est donc un phénomène $d^{\prime}$ abord naturel et indispensable à la vie terrestre.

Les GES sont naturellement très peu abondants, mais du fait de l'activité humaine la concentration de ces gaz dans l'atmosphère s'est sensiblement modifiée: ainsi, depuis 1750, année considérée comme celle du début de la "civilisation industrielle", la concentration en $\mathrm{CO}_{2}$, principal $\mathrm{GES}$, a augmenté de $30 \%$, celle de $\mathrm{CH}_{4}$ de $145 \%$ [1]. L'activité humaine a également contribué à l'émission dans l'atmosphère de nouveaux composés à effet de serre, issus de synthèses chimiques. Les principaux GES, naturels ou issus de synthèses, sont présentés dans le tableau 1.

Depuis la fin du XIX siècle, la terre s'est réchauffée de $0,6^{\circ} \mathrm{C}$ et on a assisté à une

\begin{abstract}
Whether the industry wants to anticipate the new regulations concerning the greenhouse gas (GHG) emissions, or better control the costs of fluctuating fossil energy, the Bilan Carbone ${ }^{\circledR}$ assessment is a tool available to evaluate the company's exposure, or even vulnerability, pertaining (related) to these two strategic axes for the development of corporate activity. ITERG has carried out 15 Bilan Carbone ${ }^{\circledR}$ in oils and fats industry. This method is a solution for ranking emissions source by source and a good start to launch a dynamic process to reduce these emissions. In all the cases of the study, the emissions are strongly related to raw materials, freight, packaging, and consumption of fossil energy. The reduction of $\mathrm{GHG}$ emissions, is possible with an increased utilisation of the biomass, an improved valorisation of by-products by anaerobic digestion with recovery of the produced biogas for energy needs, and when rail, canals, rivers, or seas are preferably chosen to road transport for freight. In the oleochemicals industry, GHG emissions related to the raw materials can be reduced by the development of bio-lubricants, and innovative new products formulation based on vegetable resources.

Key words: oils and fats industry, derivatives, environment, climate change, Bilan Carbone ${ }^{\circledast}$ method, greenhouse gases
\end{abstract}

montée du niveau des océans de 10 à $25 \mathrm{~cm}$. L'influence des activités humaines sur ces variations est depuis février 2007 confirmée par le GIEC, Groupe d'experts Intergouvernemental sur l'Evolution du Climat [3] : " Les concentrations mondiales actuelles de dioxyde de carbone, de méthane et de protoxyde d'azote ont crû de façon notable par suite des activités humaines depuis 1750 (...). Les augmentations du dioxyde de carbone sont principalement dues à l'utilisation des combustibles fossiles et au changement $d^{\prime} u t i l i s a t i o n$ des terres, tandis que ceux $d u$ méthane et du protoxyde d'azote sont principalement dus à l'agriculture (...). Le réchauffement du système climatique est sans équivoque, car il est maintenant évident dans les observations de l'accroissement des températures moyennes mondiales de l'atmosphère et de l'océan, la fonte généralisée de la neige et de la glace, et l'élévation du niveau moyen mondial de la mer».

A l'échelle des continents, des régions et des bassins océaniques, de nombreux changements à long terme du climat ont été observés. Ils incluent des changements de température, de volume des glaces arctiques, de quantité de précipitations, de salinité de l'océan, des structures des vents et des aspects de situations météorologiques extrêmes, comme les séche- resses, les fortes précipitations, les vagues de chaleur et l'intensité des cyclones tropicaux.

Les informations paléoclimatiques confirment I'interprétation que le réchauffement du dernier demi-siècle est atypique sur au moins les 1300 dernières années. La dernière fois que les régions polaires ont été significativement plus chaudes qu'actuellement pendant une longue durée (il y a environ 125000 ans), la réduction du volume des glaces polaires a conduit à une élévation du niveau des mers de 4 à 6 mètres. A la suite de ce constat, le GIEC dresse des perspectives alarmantes:

- la poursuite des émissions de gaz à effet de serre au niveau actuel ou au-dessus provoquerait un réchauffement supplémentaire et induirait de nombreux changements dans le système climatique global au long du XXI ${ }^{\mathrm{e}}$ siècle, qui seraient très probablement plus importants que ce qui a été observé au cours du siècle précédent :

- le réchauffement le plus important est attendu sur les terres émergées et aux latitudes élevées, et le moins important devrait apparaître dans le sud de I'Océan indien et dans certaines parties de l'Atlantique-Nord ;

- les simulations étudiées produisent une contraction de la couverture neigeuse, une
Tableau 1. Les principaux GES.

\begin{tabular}{|lc|}
\hline Composés & Ordre de grandeur de leur durée de vie dans l'atmosphère [2] \\
\hline Dioxyde de carbone $\left(\mathrm{CO}_{2}\right)$ & Le siècle \\
Méthane $\left(\mathrm{CH}_{4}\right)$ & La décennie \\
Oxyde nitreux $\left(\mathrm{N}_{2} \mathrm{O}\right)$ & Le siècle \\
Hydrofluorocarbures $\left(\mathrm{C}_{\mathrm{n}} \mathrm{H}_{\mathrm{m}} \mathrm{F}_{\mathrm{p}}\right)$ & Quelques semaines à quelques siècles \\
Hexafluorure de soufre $\left(\mathrm{SF}_{6}\right)$ & Quelques milliers d'années \\
Perfluorocarbures $\left(\mathrm{C}_{\mathrm{n}} \mathrm{F}_{2 n+2}\right)$ & Quelques siècles à plusieurs dizaines de millénaires \\
\hline
\end{tabular}


diminution de la banquise dans l'Arctique comme dans l'Antarctique; pour certaines simulations, la glace disparaît presque entièrement en Arctique à la fin de l'été dans la seconde partie du XXI ${ }^{\mathrm{e}}$ siècle ;

- il est très probable que les chaleurs extrêmes, les vagues de chaleur, et les événements de fortes précipitations seront de plus en plus fréquents;

- il est aussi probable que les cyclones tropicaux (typhons et ouragans) deviendront plus intenses, avec des précipitations et des vents plus forts :

- les projections déplacent vers les pôles les trajectoires des tempêtes non tropicales, avec des changements en conséquence sur la répartition des vents, des précipitations et des températures, dans la continuité des tendances observées lors du demi-siècle écoulé ;

- le réchauffement et l'élévation du niveau de la mer dus à l'homme continueraient pendant des siècles à cause des échelles de temps associées aux processus climatiques et aux rétroactions, même si les concentrations des gaz à effet de serre étaient stabilisées ;

- à cause du couplage entre le climat et le cycle du carbone, on s'attend à une augmentation du dioxyde de carbone dans l'atmosphère avec le réchauffement du système climatique ;

- les émissions anthropiques de dioxyde de carbone, passées et futures, continueront à contribuer au réchauffement et à l'élévation du niveau de la mer pendant plus d'un millénaire, en raison des échelles de temps nécessaires à sa disparition de l'atmosphère.

La répartition des émissions de GES en France en 2005 par secteurs [4] indique que l'industrie est responsable de $20,7 \%$ de ces émissions, derrière les transports $(26,5 \%)$, mais devant l'agriculture $(18,6 \%)$, le résidentiel et le tertiaire $(18,5 \%)$, l'énergie $(12,7 \%)$.

\section{Le protocole de Kyoto et son application}

La Convention Cadre des Nations Unies sur les Changements Climatiques (UNFCCC), adoptée à Rio en juin 1992 et aujourd'hui ratifiée par 188 pays, a reconnu l'existence du changement climatique d'origine humaine et imposé aux pays industrialisés la responsabilité de lutter contre ce phénomène. L'objectif de la convention est de stabiliser les concentrations de GES dans l'atmosphère à un niveau qui empêche toute « perturbation anthropique dangereuse du système climatique ». La convention précise que ce niveau doit être atteint dans un délai suffisant pour que les écosystèmes puissent s'adapter naturellement aux changements climatiques, que la production alimentaire ne soit pas menacée et que le développement économique puisse se poursuivre d'une manière durable.

Devant l'ampleur du phénomène, le Protocole de Kyoto, conclu en 1997, a renforcé la contrainte. II prévoit l'établissement d'un marché international d'échange de droits d'émissions de $\mathrm{CO}_{2}$ et impose pour les pays signataires de réduire globalement les émissions des six principaux GES de 5,2 \% par rapport à l'année 1990 pour la période 2008-2012.

L'Union européenne s'est engagée à une réduction de $8 \%$ de ces émissions par rapport à 1990 pour cette même période. Dans cette optique, une directive européenne de 2003 a requis de la part de chaque Etat membre l'établissement d'un plan national d'allocation de quotas d'émissions (PNAQ) de $\mathrm{CO}_{2}$ définissant les volumes d'émissions annuelles à ne pas dépasser [5]. On retrouve, parmi les activités couvertes par la directive, l'émission de $\mathrm{CO}_{2}$ par les installations de combustion d'une puissance calorifique de combustion supérieure à $20 \mathrm{Mw}$, soumises à autorisation sous la rubrique $n^{\circ} 2910$ de la nomenclature des ICPE (installations classées pour la protection de l'environnement) de la réglementation française, pour les secteurs de la chimie et de I'agroalimentaire.

En France, le $1^{\text {er }}$ plan («PNAQ I ») a couvert la période 2005-2007 [6]. Le PNAQ II couvre la période 2008-2012 [7]. Ces dispositions, actuellement limitées aux émissions provenant du secteur industriel et de la production d'énergie, risquent d'être étendues aux vols aériens dès 2011 [8].

\section{Une nécessité de savoir pour anticiper}

L'émission de gaz à effet de serre (GES) devient donc désormais une préoccupation environnementale, économique et sociétale majeure, maintenant associée à des contraintes réglementaires.

II semble vraisemblable, au vu du contexte international, qu'il sera demandé à l'avenir aux entreprises, mais aussi aux particuliers, un effort plus important de maîtrise des émissions, afin de lutter contre un changement climatique dont l'avènement ne fait aucun doute au sein de la communauté scientifique; notre seule marge de manœuvre est de le rendre moins brutal, en diminuant aussi vite que possible les émissions d'origine humaine. L'ordre de grandeur de l'effort nécessaire à l'échelle planétaire, pour arrêter d'enrichir l'atmosphère en gaz carbonique, est une division par 2 des émissions anthropiques, ce qui implique, si le droit à émettre est le même pour tout terrien, une division par 4 des émissions par personne en France d'ici 2050 [9] !
Dans ce contexte, il est aujourd'hui nécessaire de connaître au mieux les émissions de GES générées par l'activité industrielle, en identifiant les différents postes d'émissions, pour ébaucher des solutions visant aussi bien à réduire ces émissions qu'à maîtriser l'énergie, et par conséquent réduire la dépendance des entreprises vis-à-vis des combustibles fossiles.

En effet, un site industriel (ou toute entreprise ou collectivité) est " émetteur » à plus d'un titre :

- par ses émissions directes, issues des combustions en chaudières par exemple ;

- par les procédés utilisés (la fuite des fluides frigorigènes des systèmes de refroidissement) ; - par l'utilisation de matériaux ou produits dont la fabrication a engendré des émissions ; - par les transports induits: livraison des matières premières et réactifs, expédition des produits finis, déchets... ;

- par les déplacements de personnes : salariés entre leur domicile et le lieu de travail, les déplacements professionnels, les visiteurs, clients... ;

- par l'usage qu'il sera fait des produits ou services vendus, mais aussi par le traitement des déchets générés ;

- par toute autre source parfois insoupçonnée...

Il convient donc d'évaluer l'impact de ces différents postes afin d'estimer la vulnérabilité de son activité face à de fortes contraintes réglementaires futures, une importante augmentation du coût des énergies fossiles, ou une impossibilité de s'approvisionner en celles-ci.

Dans cette optique, I'ADEME propose un outil, le Bilan Carbone ${ }^{\circledR}$, qui permet de comptabiliser les émissions de GES de toute entreprise ou collectivité, par l'analyse des différentes sources. La méthode conduit à ébaucher des solutions visant aussi bien à réduire ces émissions qu'à maîtriser l'énergie, et par conséquent réduire la dépendance des entreprises vis-à-vis des combustibles fossiles.

Les objectifs de la méthode sont de :

- calculer des émissions de carbone générées par les activités d'une entreprise ;

- hiérarchiser le poids des émissions en fonction des activités ;

- situer l'impact de ses activités en matière de GES ;

- définir des préconisations pour réduire ces émissions.

Les résultats du bilan permettent :

- d'évaluer les consommations énergétiques des activités ;

- de mettre en place une comptabilité des émissions de GES ;

- de bénéficier d'un nouvel indicateur de performance dans le cadre du management environnemental : le Bilan Carbone ${ }^{\circledR}$ peut donner un second souffle à un SME (système de 
management environnemental) en panne d'inspiration face à la contrainte d'amélioration continue... :

- de bénéficier d'un outil d'aide à la décision ; - de s'interroger sur les choix logistiques;

- de disposer de critères de choix des fournisseurs ;

- de répondre aux exigences réglementaires, ou de les anticiper ;

- d'exploiter les résultats dans le cadre de la politique de communication de l'entreprise ; - d'intégrer l'entreprise dans une démarche de développement durable, par son implication dans la lutte contre le changement climatique.

L'ISO a publié une série de normes relatives à la quantification des émissions de GES [10-12]. Elles donnent des spécifications et lignes directrices, sans préciser pour autant une méthodologie particulière. La première norme précise seulement que « I'organisme doit sélectionner et utiliser des méthodologies de quantification qui réduiront raisonnablement l'incertitude et donneront lieu à des résultats exacts, cohérents et reproductibles». La méthode Bilan Carbone ${ }^{\circledR}$ fait partie des types de méthodologies retenues par la norme (" calcul fondé sur les données d'activité GES multipliées par les facteurs d'émission ou de retrait de GES ») et permet de présenter les résultats en fonction des périmètres opérationnels décrits par la norme :

- émissions directes de GES : celles provenant des installations situées à l'intérieur des périmètres organisationnels de l'organisme ;

- émissions de GES à énergie indirecte : celle provenant de la production d'électricité, de chaleur ou de vapeur importée pour son usage ;

- autres émissions indirectes de GES.

Il existe différentes méthodologies de comptabilisation des émissions de GES [13], mais aucune ne semble pouvoir intégrer de manière aussi transversale les différents postes d'émission des activités d'une entreprise.

\section{Des opérations groupées}

Afin de sensibiliser les professions des corps gras aux risques liés au changement climatique, I'ITERG s'est engagé avec I'ADEME dans des opérations groupées visant à réaliser des Bilans Carbone ${ }^{\circledR}$ sur des sites industriels. La première opération, appelée GESSICA ${ }^{1}$, nous a permis de réaliser 5 Bilans Carbone ${ }^{\circledR}$. La

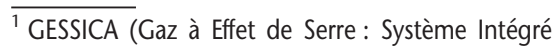
pour la Comptabilisation dans les Industries Alimentaires) a reçu le soutien financier de l'ADEME et de I'ONICC et a permis à 6 Centres techniques de I'agro-alimentaire de réaliser 30 Bilans Carbone ${ }^{\circledR}$.
}

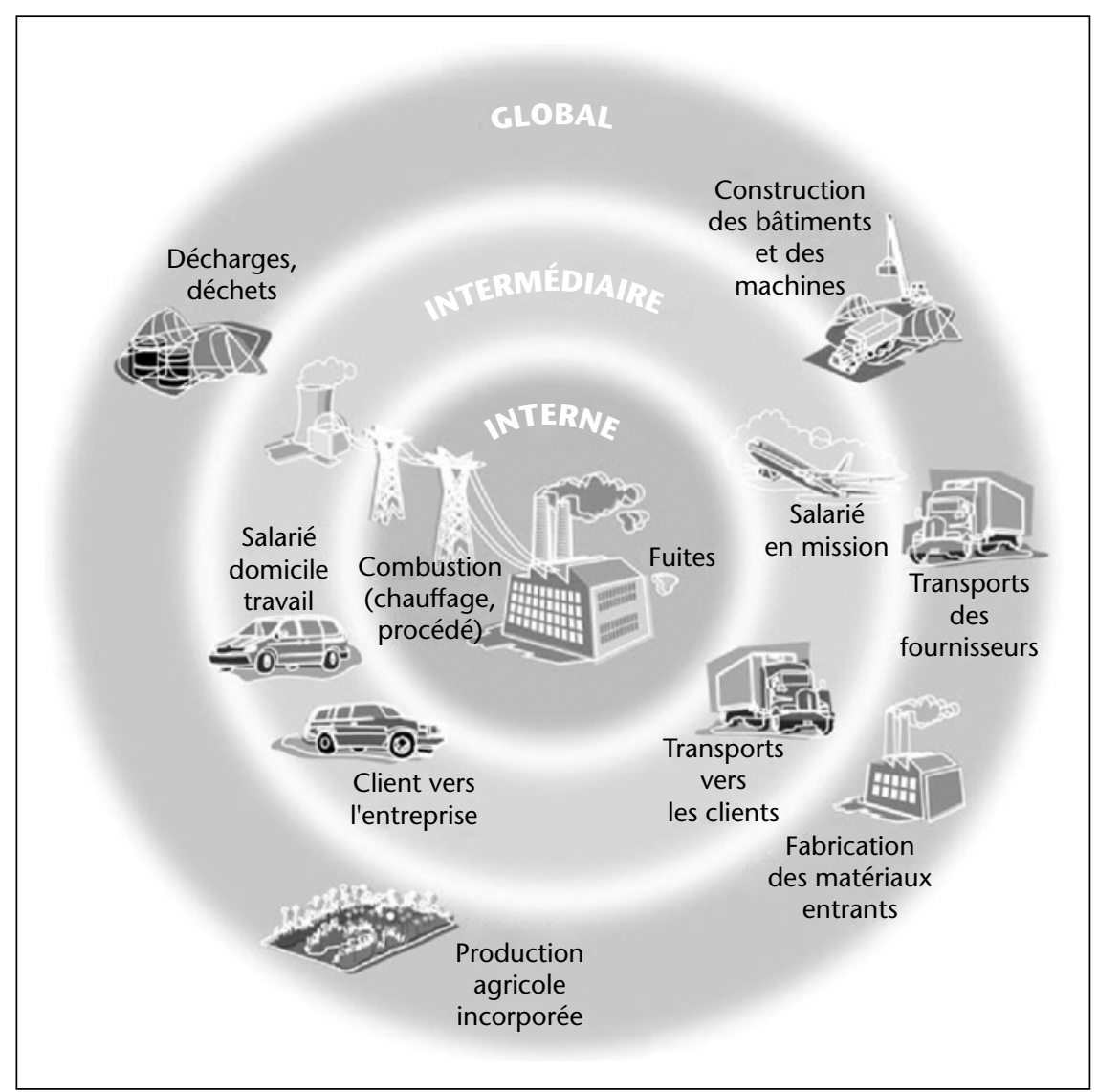

Figure 1. Les périmètres $d^{\prime}$ investigation de la méthode Bilan Carbone ${ }^{\circledR}$.

seconde opération, coordonnée par le Réseau $\mathrm{CTI}^{2}$, nous a permis d'en réaliser 10 .

L'ITERG a donc pu appliquer cette méthode sur 15 sites, couvrant les différents sous secteurs de l'industrie des corps gras : 4 sites de transformation de sous-produits d'origine animale, 2 sites de trituration de graines oléagineuses, 2 sites de raffinage et de conditionnement d'huiles, 2 sites de trituration et de raffinage, 2 sites de trituration, raffinage et estérification, 3 sites de lipochimie.

\section{Méthodologie employée}

Les émissions de GES du site ont été évaluées en utilisant la version V 3.3 de la méthode Bilan Carbone ${ }^{\circledR}$ développée par I'ADEME. Cette méthode passe en revue tous les flux physiques qui concernent l'entreprise (flux de personnes, d'objets, d'énergies, de matières premières...), et leur fait correspondre les émissions de GES qu'ils engendrent (figure 1). Ces émissions sont agrégées poste par poste : transports de mar-

\footnotetext{
${ }^{2}$ Ce programme, financé par l'ADEME, a permis à 11 Centres Techniques Industriels de réaliser 68 Bilans Carbone $^{\circledR}$.
}

chandises par route, combustibles utilisés en interne...

S'il est courant de mesurer la concentration en GES dans l'air, les émissions ne peuvent faire l'objet d'une mesure directe qu'à titre exceptionnel. La seule manière d'estimer ces émissions est alors de les obtenir par le calcul, à partir d'autres données, et la méthode Bilan Carbone ${ }^{\circledR}$ a précisément été fondée sur cette approche: les données liées à l'activité de I'entreprise sont converties en émissions de GES, exprimées en équivalent carbone, en leur appliquant un facteur d'émission.

L'équivalent carbone est la mesure " officielle » des émissions de gaz à effet de serre. Cependant, beaucoup d'entreprises préfèrent utiliser «l'équivalent $\mathrm{CO}_{2}$ " (donnant des valeurs 3,67 fois supérieures, dans un rapport de 44/12, correspondant au rapport des masses moléculaires du $\mathrm{CO}_{2}$ et du carbone).

Les différents postes de l'activité de l'entreprise pris en compte sont :

- l'énergie interne : les émissions liées à l'utilisation de l'énergie sur le site,

- l'énergie sous-traitant: émissions liées à I'utilisation de l'énergie pour les procédés soustraités, 
- les procédés internes : émissions des procédés industriels (hors usage de l'énergie), - les procédés des sous-traitants : émissions des procédés industriels (hors usage de l'énergie) pour les procédés sous-traités,

- le fret, correspondant aux émissions liées au transport des marchandises: fret interne, fret des produits finis, fret des matières entrantes (tous types de fournisseurs),

- le transport des personnes: déplacements domicile-travail des salariés, déplacements professionnels, déplacements des "visiteurs", - les matériaux et services entrants : émissions liées aux matériaux entrants et services tertiaires,

- les déchets : émissions liées aux déchets solides et eaux usées,

- les emballages: émissions liées au traitement de fin de vie des emballages,

- les amortissements : émissions liées à l'amortissement des immobilisations (bâtiments, parkings et voies d'accès, matériel informatique, équipements, véhicules, mobiliers, autres biens...).

La méthode propose plus de 500 facteurs d'émission. Cependant, dans certains cas, nous avons fait le choix d'utiliser des facteurs autres que ceux proposés par la méthode. Par exemple, la méthode donne un facteur d'émission pour «I'huile de tournesol» de $917 \mathrm{~kg}$ éq $\mathrm{CO}_{2} / \mathrm{t}$. Le calcul de cette valeur n'est pas détaillé; nous supposons qu'il s'agit d'une huile raffinée, conditionnée, et acheminée jusqu'au consommateur. Nous avons préféré utiliser des facteurs issus d'une étude relative aux filières de production de biocarburants [14]. Nous avons donc retenu dans les Bilans Carbone ${ }^{\circledR}$ que nous avons réalisés un facteur de $598 \mathrm{~kg}$ éq CO $/ \mathrm{t}$ pour la graine de colza, et de $433 \mathrm{~kg}$ éq $\mathrm{CO}_{2} / \mathrm{t}$ pour la graine de tournesol. Ces facteurs prennent en compte l'usage des engrais azotés et des produits phytosanitaires pour la culture de la graine, mais aussi la mécanisation (consommation du tracteur et surconsommation liée aux différents passages d'outils tractés sur les sols cultivés).

Dans le cas du colza, les émissions de GES sont liées à près de $55 \%$ à celles du $\mathrm{CO}_{2}$ et à plus de $40 \%$ à celles du $\mathrm{N}_{2} \mathrm{O}$. Dans le cas du tournesol, les émissions sont liées à près de $75 \%$ au $\mathrm{CO}_{2}$ et à plus de $20 \%$ au $\mathrm{N}_{2} \mathrm{O}$.

Par sa transversalité, la comptabilisation des émissions de GES offre un nouveau regard sur l'activité de l'entreprise et permet de garantir, ou d'évaluer, sa pérennité. De plus, la nouvelle version de la méthode inclut un modèle de calcul économique permettant de simuler une hausse du coût de l'énergie ou l'entrée en vigueur d'une "taxe carbone». Elle permet également de comparer deux projets : le choix du lieu d'implantation d'une nouvelle usine en fonction des contraintes de fret, le choix des matériaux de construction, des filières de traitements des déchets qui seront générés...

\section{Résultats et discussion}

Nous présentons les résultats obtenus sur trois sites industriels :

- le site de Saipol à Grand Couronne (SeineMaritime - France), produisant des huiles végétales et des esters méthyliques ;

- le site de France Gras à Le Sourn (Morbihan France), exerçant une activité de collecte, dépôt et transformation en farines et graisses de sous-produits d'origine animale ;

- le site de Lixol (Gironde - France), produisant des résines synthétiques alkydes à base d'huiles végétales.

\section{Les émissions de GES de I'usine Saipol à Grand Couronne}

Les activités de l'usine de Grand Couronne sont centrées sur deux pôles de fabrication :

- la trituration des graines oléagineuses pour la production d'huiles et de tourteaux ;

- la fabrication de Diester et de glycérine.

Les émissions liées à l'activité globale de I'année 2005 représentent 732347 tonnes équivalent $\mathrm{CO}_{2}$ (figure 2). Ce bilan est impacté à près de $70 \%$ par les matières premières traitées (graines de colza, huiles brutes) en raison des tonnages importants et des facteurs d'émission élevés pour les produits d'origine agricole ; en effet, le facteur d'émission prend en compte toute l'activité nécessaire à la culture des graines oléagineuses.

L'exploitant n'ayant que peu de maîtrise sur la nature de la matière première, son extraction du bilan permet de considérer l'activité spécifique du site industriel, qui ne représentent plus alors que 191046 t éq. $\mathrm{CO}_{2}$, et qui se répartissent de la manière suivante : $44 \%$ liés au fret routier, $39 \%$ liés au gaz alimentant la chauffe- rie interne et celui nécessaire à la production de vapeur par la cogénération, $10 \%$ liés aux consommables, $3 \%$ liés au fret fluvial, $2 \%$ liés aux procédés internes, $2 \%$ liés à l'électricité, $1 \%$ liés aux services.

Les autres sources d'émissions (amortissement, traitement des déchets, déplacements des personnes, fret ferroviaire) sont inférieures à $1 \%$ du bilan du site (sans les matières premières). Cette répartition est présentée sous forme graphique figure 3.

\section{Le fret}

Le fret pris en compte est celui des matières premières (graines oléagineuses, huiles brutes), des consommables (hexane pour l'extraction, acides, soude et terre de décoloration pour le raffinage, méthanol et méthylate de sodium pour l'estérification).

Les graines sont réceptionnées par camion (70 \% du tonnage des graines), train ( $24 \%$ du tonnage) ou péniche (6\% du tonnage). Les huiles brutes et les consommables sont réceptionnés par camion.

Le fret comprend également l'expédition des produits finis: les huiles raffinées sont expédiées par camion, les esters par bateau (64\% du tonnage des esters), train ( $28 \%$ du tonnage) ou camion (8 \% du tonnage) ; les huiles brutes sont expédiées par bateau; les tourteaux et autres co-produits (pâtes de neutralisation, huiles acides, glycérine) sont expédiés par camion.

Le fret routier représente $93 \%$ des émissions du fret, pour seulement $73 \%$ du tonnage transporté. Le fret ferroviaire ne représente que $1 \%$ des émissions, pour $14 \%$ du tonnage. Le fret maritime et fluvial ne représente que $6 \%$ des émissions de GES, pour $14 \%$ du tonnage transporté.

Les matières, peu altérables, sont adaptées à des transports ferroviaire, maritime ou fluvial.

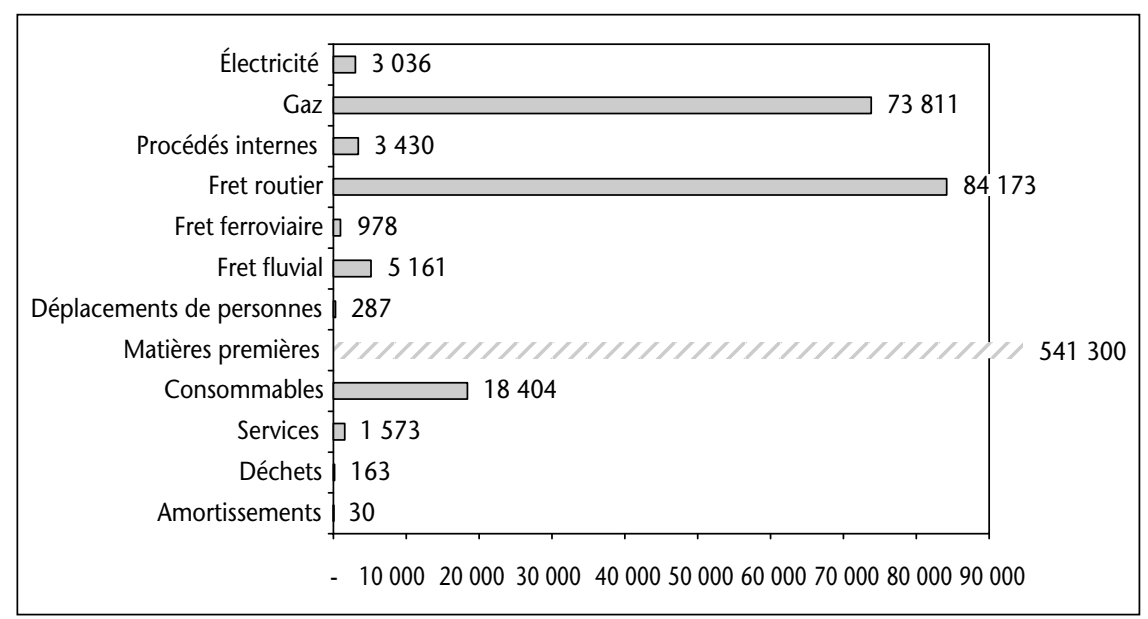

Figure 2. Bilan global récapitulatif des émissions de GES du site de Saipol à Grand Couronne (en téq $\mathrm{CO}_{2}$ ). 


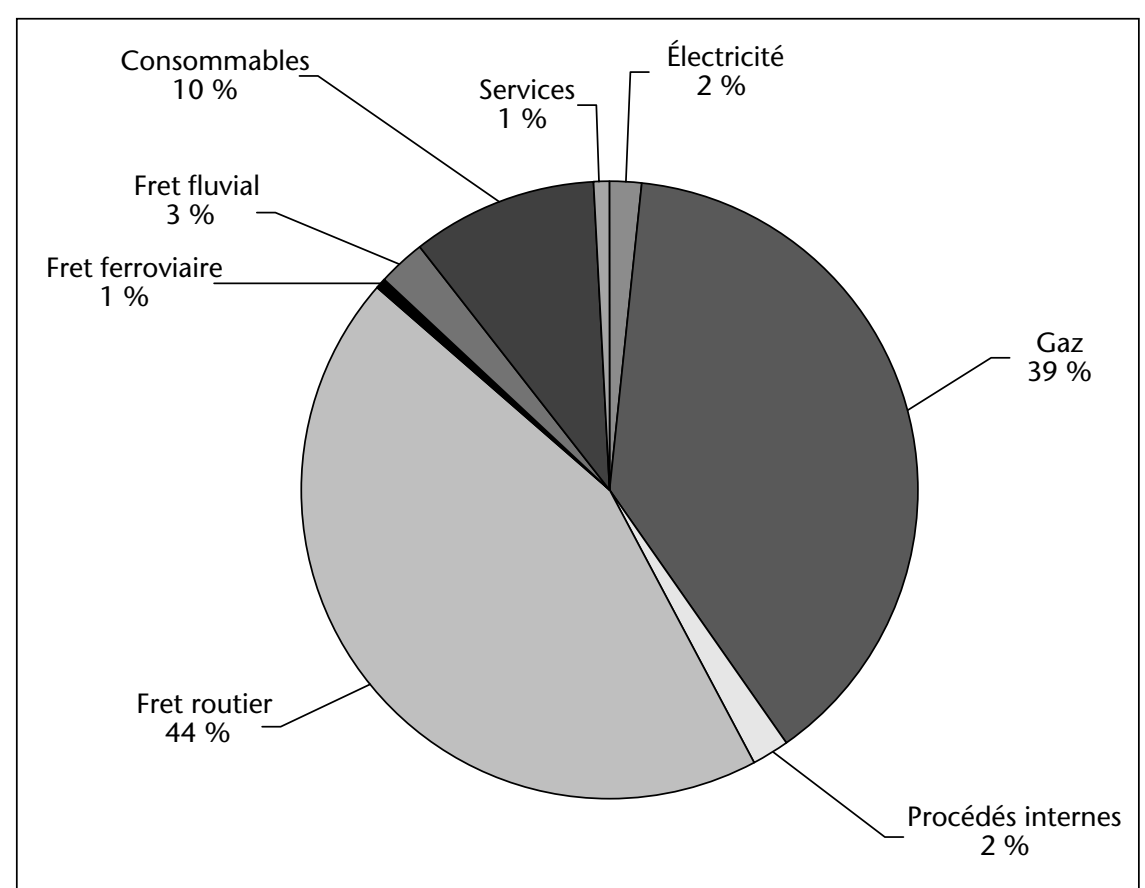

Figure 3. Répartition des émissions de GES du site de Saipol à Grand Couronne (sans les matières premières).

Ces types de transport, déjà pratiqués pour certaines matières, peuvent être développés. Pour information, une tonne de marchandise transportée par camion sur $1 \mathrm{~km}$ représente une émission de $115 \mathrm{~g}$ de $\mathrm{CO}_{2}$. Cette tonne, transportée sur $1 \mathrm{~km}$ par un train en France, ne représente plus qu'une émission de $11 \mathrm{~g}$ de $\mathrm{CO}_{2}$, c'est-à-dire 10 fois moins !

\section{La consommation de gaz}

Les besoins en vapeur du site sont importants : $39 \%$ des émissions de GES liées à l'activité du site (sans tenir compte des matières premières) sont dus au gaz consommé directement par les chaudières internes de l'usine, ou via une cogénération dont l'exploitation est externalisée. L'électricité produite en France est majoritairement d'origine nucléaire et ne génère donc que très peu d'émissions de GES : les consommations de gaz (directes ou via la cogénération) représentent $96 \%$ des émissions d'énergies internes.

Les axes de réduction de ce poste sont la réalisation d'un diagnostic énergétique, afin d'identifier les possibles réductions de consommations, mais surtout l'étude des possibilités de remplacement de l'énergie fossile par des biocombustibles (corps gras) ; plus spécifiquement, le traitement des sous-produits du raffinage (terres usagées; pâtes de neutralisation) par méthanisation sur le site même aurait le double avantage de produire un biogaz disponible pour les besoins du site, mais aussi de s'affranchir de l'activité de l'atelier de " cassage des pâtes de neutralisation ", activité contrai- charge organique élevée) au profit d'un traitement par méthanisation.

\section{Bilan par activité}

Nous avons réparti les émissions de GES (sans tenir compte de la contribution des matières premières : graines et huiles brutes réceptionnées) en fonction des trois activités du site. Les émissions sont liées :

- à $59 \%$ à la trituration, principalement en raison du fret et des consommations de gaz pour la production de vapeur ;

- à $27 \%$ à l'estérification, principalement en raison des consommations de gaz et de consommables ;

- à $14 \%$ à l'activité de raffinage (et de semiraffinage).

\section{Les émissions de GES de France Gras à Le Sourn}

Les émissions globales du site de France Gras à Le Sourn représentent 47853 t éq $\mathrm{CO}_{2}$ pour I'année 2006, et sont liées à $90 \%$ aux matières premières collectées (figure 4), en raison du tonnage important mais aussi des facteurs d'émission très élevés pour les produits d'origine animale : le facteur associé aux produits porcins est de $4143 \mathrm{~kg}$ éq $\mathrm{CO}_{2} / \mathrm{t}$ !

gnante, présentant des risques pour le personnel (utilisation d'acides à température élevée) et consommatrice d'énergie. Une étude est actuellement en cours à I'ITERG sur ce sujet.

\section{Les consommables}

L'ensemble des consommables (hexane pour I'extraction ; acides, soude et terre de décoloration pour le raffinage; méthanol et méthylate de sodium pour l'estérification...) représente des quantités importantes. Le process étant optimisé, les axes de réduction de leur consommation sont limités. Bien que déjà important, l'impact des consommables est sous-estimé, car il n'a pas été possible d'attribuer à chacun un facteur d'émission, par manque de données disponibles (notamment pour les terres de décoloration).

\section{Les procédés internes}

Ce poste prend en compte les fuites de fluide frigorigène (R $134 \mathrm{a}$ ) du groupe froid du raffinage alimentaire et les émissions liées à l'activité de la station d'épuration aérobie de traitement des effluents aqueux.

Les fuites de fluides frigorigènes peuvent être prévenues par une surveillance du circuit et la mise en place d'une procédure favorisant l'intervention rapide de l'équipe de maintenance en cas de détection.

La charge organique et le volume des effluents bruts peuvent être réduits en abandonnant le traitement des pâtes par "cassage » (atelier générateur d'effluents aqueux ayant une
L'extraction de la contribution des matières collectées au bilan permet de mettre en évidence celle des autres postes. Le bilan est alors de 4721 t éq $\mathrm{CO}_{2}$, et est lié à $87 \%$ au fret, à $8 \%$ aux énergies internes, à $3 \%$ aux effluents aqueux. Les autres postes sont inférieurs à $2 \%$. Cette répartition est présentée sous forme graphique figure 5 .

\section{L'énergie} l'électricité, de la vapeur achetée à l'usine d'incinération d'ordures ménagères voisine et de la vapeur produite par la chaufferie du site à partir de graisse animale (ou de fuel lourd, mais en très faible quantité). L'alimentation en vapeur par l'usine voisine et l'utilisation des graisses permettent de réduire les émissions de GES liées aux énergies internes. tion d'un diagnostic énergétique et la poursuite du remplacement du fuel par des graisses animales.

L'ITERG a récemment réalisé une étude sur le traitement par méthanisation des eaux gélatineuses du site de Le Sourn. Celles-ci sont issues de la fonte des corps gras animaux par voie humide. Le site en génère environ $40000 \mathrm{t} / \mathrm{an}$, qui sont actuellement concentrées sur un évaporateur 4 effets, avant d'être incinérées en externe, à un coût élevé. Après des essais de laboratoire et la mise en fonctionnement $d^{\prime}$ un pilote de méthanisation (de $2 \mathrm{~m}^{3}$ ) pendant 4 mois sur le site de France Gras, nous avons
L'énergie utilisée en interne est constituée de

Les axes de réduction identifiés sont la réalisa- 


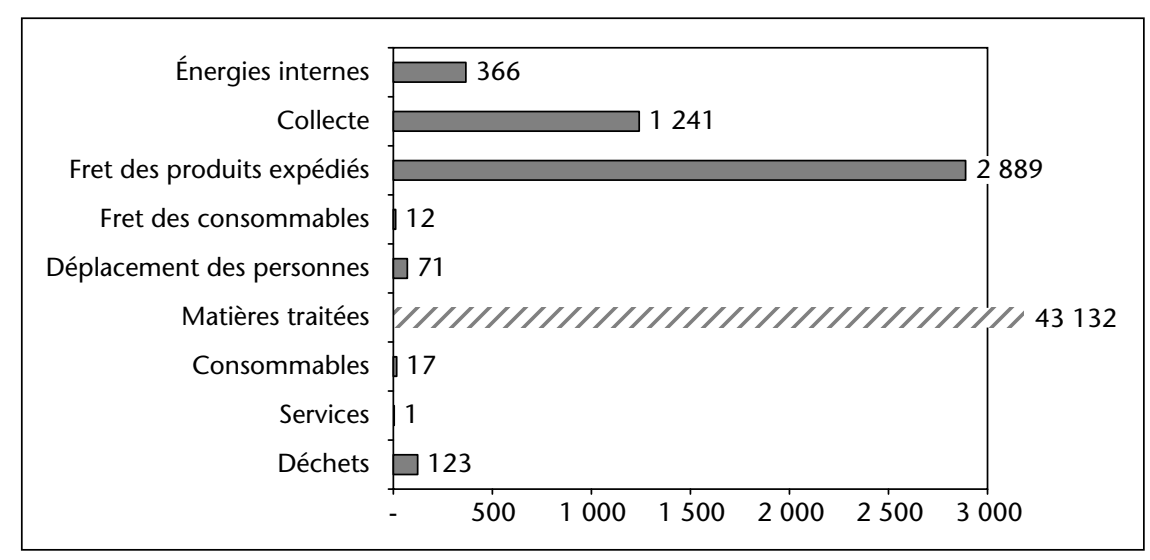

Figure 4. Bilan global récapitulatif des émissions de GES du site de France Gras (en téqu. $\mathrm{CO}_{2}$ ).

démontré la faisabilité de méthaniser ces eaux dans l'unité de production de corps gras animaux.

Cette étude prouve que la biodégradabilité des eaux gélatineuses en digestion anaérobie est très élevée (supérieure à $98 \%$ ) et que la quantité de biogaz générée est très importante : elle est de l'ordre de $0,7 \mathrm{~m}^{3} / \mathrm{kg}$ de DCO éliminée [15]. Les premières estimations indiquent que l'énergie produite pourrait permettre de couvrir environ $30 \%$ des besoins énergétiques actuels du site [16]. La méthanisation des eaux gélatineuses avant concentration aurait aussi l'avantage de s'affranchir de l'étape de concentration sur l'évaporateur 4 effets, très consommateur d'énergie et de temps (environ 2 équivalents-temps-plein).

\section{Le fret}

Le fret est exclusivement effectué en camion. II est composé de la collecte ( $26 \%$ des émissions de l'activité du site), de l'expédition des graisses, farines et des eaux gélatineuses concentrées (respectivement 20,14 et $7 \%$ ), mais aussi de la redistribution d'une partie de la collecte vers d'autres sites (18\%). Le fret des consommables est également pris en compte. La mise en place d'une unité de traitement des eaux gélatineuses par méthanisation sur le site permettrait également de s'affranchir du fret lié à l'expédition des eaux concentrées.

Compte tenu de la diversité des points de collecte et de la nature des sous-produits collectés (rapidement altérables), il est difficile $d^{\prime}$ envisager un autre moyen de transport que la

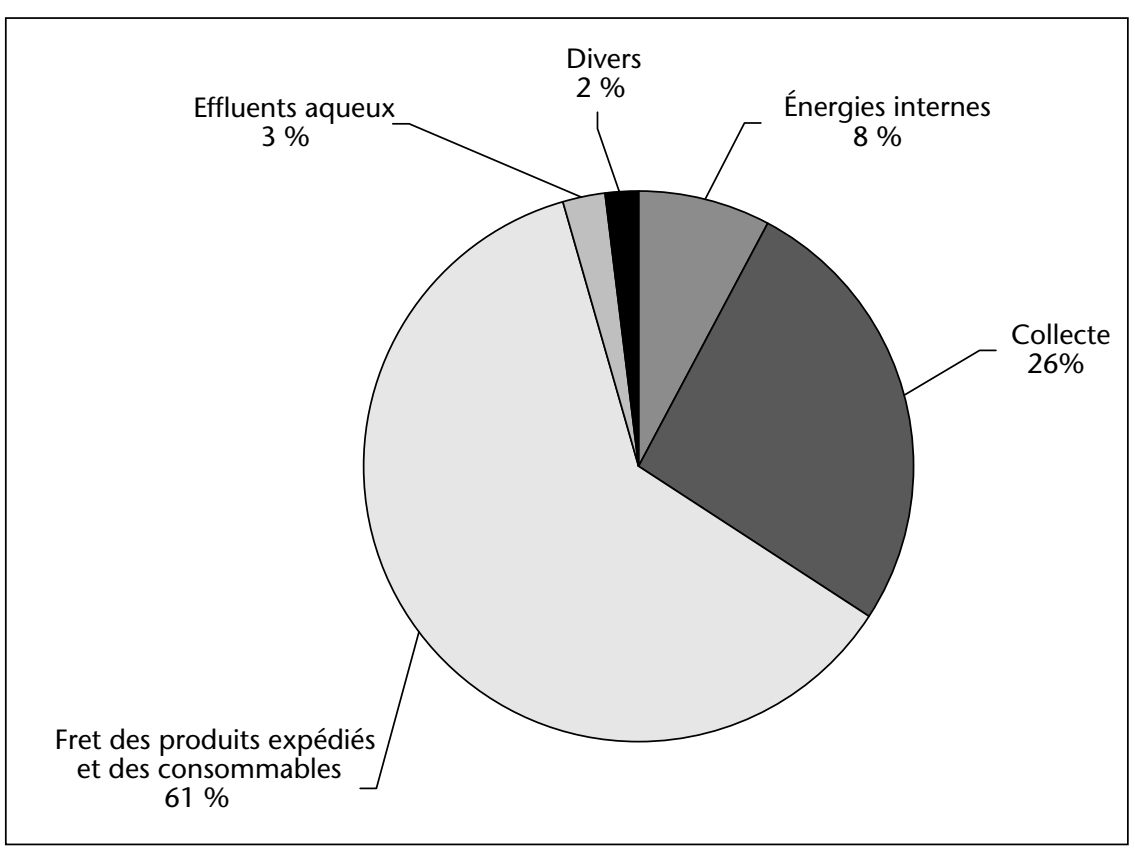

Figure 5. Répartition des émissions de GES du site de France Gras (sans les matières premières). voie routière. Les actions d'amélioration sont donc les règles de bonnes pratiques relatives au transport routier :

- optimisation des charges,

- limitation de la vitesse des véhicules,

- intégration des données de trafic (embouteillages),

- choix des véhicules (aérodynamisme, électronique moteur); adaptation du véhicule à I'utilisation (puissance du moteur),

- optimisation des itinéraires,

- formation et sensibilisation des conducteurs (mise en place d'indicateur du rendement du carburant sur le tableau de bord ou d'un dispositif de surveillance à bord de tous les tracteurs); mise en place d'un programme d'encouragement des conducteurs à l'économie (primes),

- vérification régulière de la pression des pneus,

- réduction du temps de ralenti (programmation du moteur pour qu'il s'arrête automatiquement après quelques minutes de ralenti),

- entretien régulier des véhicules,

- téléchargement des données provenant des moteurs afin d'identifier des axes de réduction des consommations.

Ces bonnes pratiques peuvent permettre une réduction des émissions de 10 à $18 \%$ [17]. Une charte d'engagements volontaires de réduction des émissions de $\mathrm{CO}_{2}$ dans le transport routier de marchandises a été élaborée par le ministère chargé des transports [18], et signée en mars 2007 par 6 entreprises; elle propose une démarche en 4 axes : moderniser la flotte de véhicules pour moins consommer, suivre efficacement la consommation de carburant pour réduire les coûts et limiter les émissions de $\mathrm{CO}_{2}$, former les conducteurs à l'écoconduite pour les responsabiliser face à leur environnement, organiser les flux de transport par l'exploitation des outils d'aide à la circulation et à la gestion de la flotte.

\section{Effluents aqueux}

La charge organique rejetée par les effluents aqueux est importante, en raison du volume annuel rejeté (environ $60000 \mathrm{~m}^{3} / \mathrm{an}$ ) et de la concentration en $\mathrm{DBO}_{5}$ (environ $1300 \mathrm{mg} / \mathrm{L}$ ). Cette charge correspond à une émission de GES de 123 t éq $\mathrm{CO}_{2} /$ an. La mise en place de I'unité de méthanisation aurait aussi pour avantage de réduire la charge organique de l'effluent aqueux en sortie de site.

\section{Les émissions de GES de Lixol}

Les émissions globales pour l'année 2005 de l'entreprise Lixol représentent 3622 t éq. $\mathrm{CO}_{2}$ (figure 6).

Ce bilan est très fortement lié :

- aux matières premières utilisées : $62 \%$ des émissions globales ; 
- à la fabrication et à la fin de vie des emballages : $20 \%$ des émissions globales (seulement $0,2 \%$ des émissions sont liées à la fin de vie); - au fret : $10 \%$ des émissions globales ;

- aux consommations d'énergie: $6 \%$ des émissions globales;

- aux déplacements des personnes : $1 \%$ des émissions globales.

Les autres postes sont inférieurs à $0,2 \%$ des émissions globales. Cette répartition est présentée sous forme graphique figure 7 .

\section{Les matières premières}

Les produits et réactifs utilisés sur le site sont divers : white spirit, acides gras, huiles, résines, xylène, anhydride phalique... Ils représentent $62 \%$ des émissions globales de l'activité du site, en raison des quantités importantes mises en jeu et des facteurs d'émission parfois élevés qui y sont associés. Les émissions de GES liées aux matières premières peuvent être réduites par une réduction des quantités de matières $d^{\prime}$ origine pétrolière au profit de matières d'origine végétale. Dans cette optique, LIXOL s'est engagé dans le programme OLEOMA, piloté par l'ITERG et relatif à la transformation industrielle des ressources oléoprotéagineuses pour des technologies respectueuses de l'environnement ; l'étude a permis de mettre au point des formulations de produits écocompatibles à faible quantité de solvant volatil [19].

\section{Les emballages}

Les emballages représentent $20 \%$ des émissions globales et $24 \%$ des émissions liées aux matières entrantes, pour seulement $7 \%$ du tonnage entrant. Cela est dû aux facteurs d'émission élevés associés à ces produits (3667 kg éq $\mathrm{CO}_{2} / \mathrm{t}$ pour le métal ; $4400 \mathrm{~kg}$ éq $\mathrm{CO}_{2} / \mathrm{t}$ pour le polyéthylène). Les émissions liées aux emballages peuvent être réduites par le choix de matériaux recyclés et la mise en place de démarches de réduction du poids des emballages.

\section{Le fret}

Les différentes matières entrantes (matières premières et produits chimiques, conditionnements : fûts métalliques, conteneurs en polyéthylène) sont transportées : à $56 \%$ en vrac, par

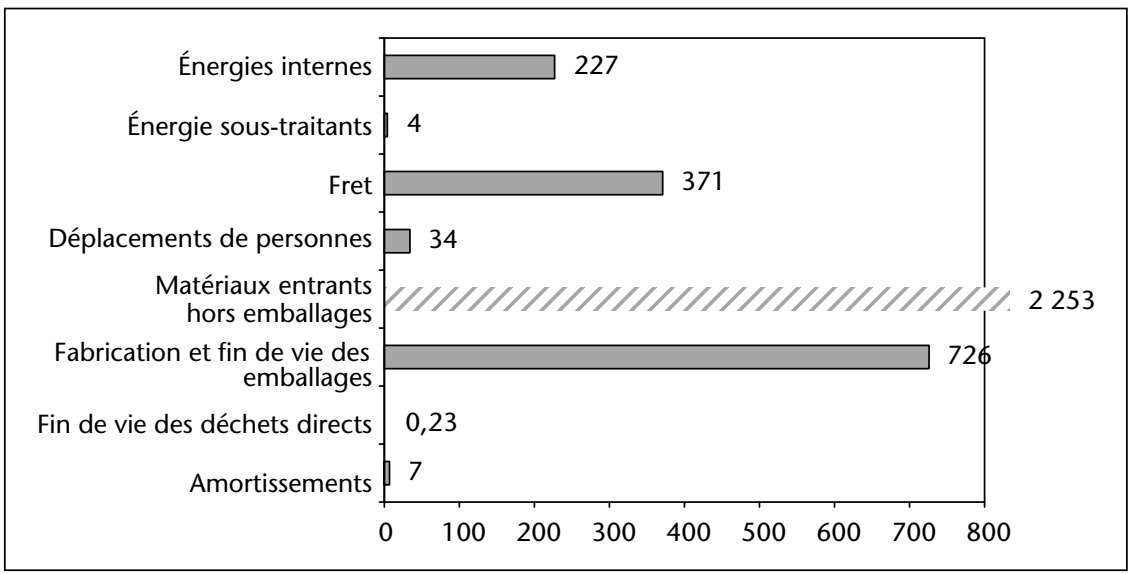

Figure 6. Bilan global récapitulatif de Lixol (t éq. $\left.\mathrm{CO}_{2}\right)$.

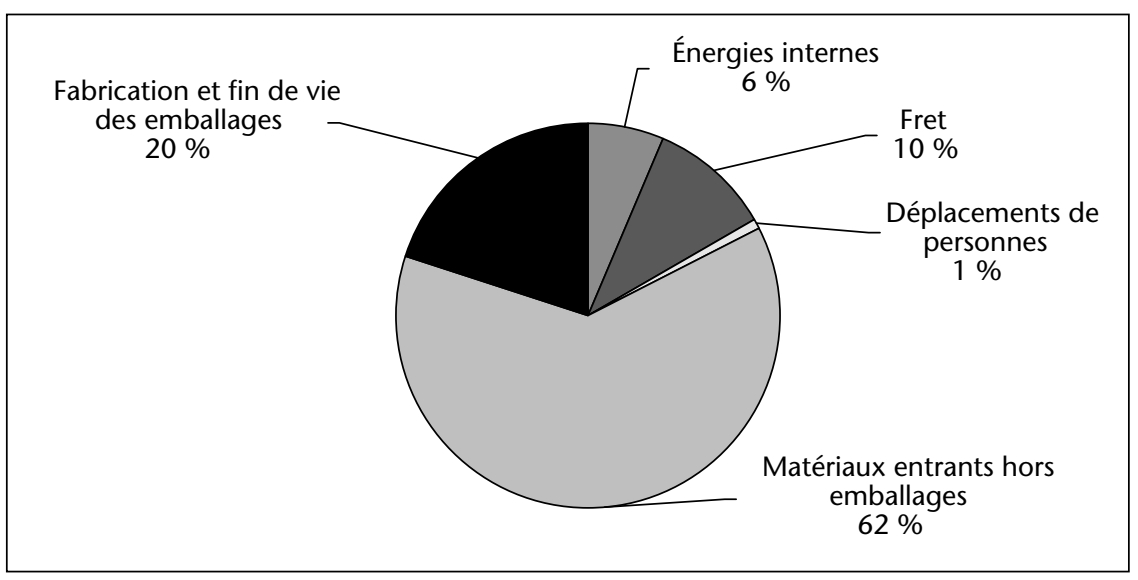

Figure 7. Répartition des émissions de GES du site de Lixol. camion; à $10 \%$ en vrac, par bateau depuis l'étranger ; à $24 \%$ par camion complet depuis divers pays européens (en sacs de $25 \mathrm{~kg}$ sur palettes ou big-bag de $500 \mathrm{~kg}$ ); à $10 \%$ par camion non dédié (218 livraisons de $25 \mathrm{~kg}$ à $14 \mathrm{t})$.

Les produits finis sont transportés : à $37 \%$ en vrac, par camion citerne vers différentes régions de France ; à $55 \%$ en fûts, par transport dédié, en France ; à $7 \%$ en containers par transport maritime (les containers sont préalablement acheminés par camion jusqu'au port du Verdon ou jusqu'à Marseille); le transport de petits colis (de 1 à $5 \mathrm{~kg}$ ) par messagerie dans toute la France n'a pas été pris en compte (environ 4 envois par mois).

Le fret routier représente $96 \%$ des émissions du fret global. Le transport maritime permet de réduire ces émissions, mais induit cependant nécessairement des fortes émissions liées au transport routier entre le lieu de production et le port d'expédition.

\section{Consommations d'énergie}

L'énergie utilisée en interne est constituée de l'électricité, du gaz propane pour l'alimentation des deux chaudières et du fuel domestique (pour le chauffage des locaux). Les émissions de ce poste ont majoritairement pour origine le gaz $(91 \%$ des émissions de ce poste). Une étude visant à réduire cette consommation est actuellement en cours sur le site.

\section{Déplacements des personnes}

La majorité des émissions (47\%) de ce poste est due aux déplacements domicile/travail des six salariés de l'entreprise. Le site étant situé en zone rurale, les transports en commun sont peu développés. Seulement un employé déjeune sur le lieu de travail. L'aménagement des locaux disponibles pourrait inciter le personnel à déjeuner sur place.

\section{Conclusion}

Une étude comparative des résultats obtenus sur les 15 sites industriels peut nous permettre de dégager certaines tendances. Cependant, la comparaison de données issues de différents Bilan Carbone ${ }^{\circledR}$ doit être effectuée avec une grande prudence et trouve rapidement ses limites, pour trois raisons principales:

- les résultats obtenus comportent une incertitude notable : elle est en moyenne de $30 \%$ sur les résultats globaux, incluant l'incertitude sur les facteurs d'émission et celle liée aux données recueillies sur le site industriel ;

- il est rare que les activités de deux sites comparés soient exactement identiques: les proportions des différentes matières traitées 
varient d'un site à l'autre (les facteurs d'émissions ne sont pas les mêmes pour la graine de colza et celle de tournesol, tout comme les facteurs des sous-produits porcins et bovins sont différents), les capacités des activités peuvent varier (un site fera plutôt du semi-raffinage que du raffinage, un autre transformera également des sous-produits animaux collectés par une entreprise extérieure, ou à l'inverse collectera des sous-produits pour le compte d'un autre site de transformation)... ;

- les périmètres d'investigation de la méthode sont rarement exactement les mêmes : ils sont fonction des données disponibles ; par exemple, dans certains cas, I'amortissement des bâtiments et du matériel a été pris en compte ; dans d'autres cas, ces informations n'étaient pas suffisamment précises pour être exploitées.

Néanmoins, en tenant compte de ces réserves, il est possible de déterminer un ordre de grandeur des émissions de GES selon l'activité du site (ces valeurs ont été déterminées sans tenir compte de la contribution des matières premières) :

- les sites de traitement de sous-produits d'origine animale génèrent de 80 à $130 \mathrm{~kg}$ éq $\mathrm{CO}_{2} / \mathrm{t}$ de matières premières; un des sites étudiés génère environ $250 \mathrm{~kg}$ éq $\mathrm{CO}_{2} / \mathrm{t}$ de matières premières, en raison de l'usage important du fuel lourd pour alimenter la chaudière ;

- les sites de trituration de graines oléagineuses génèrent environ $150 \mathrm{~kg}$ éq $\mathrm{CO}_{2} / \mathrm{t}$ de matières premières ;

- les sites de raffinage et de conditionnement $\mathrm{d}$ 'huiles végétales génèrent environ $300 \mathrm{~kg}$ éq $\mathrm{CO}_{2} / \mathrm{t}$ de matières premières ;

- les sites de trituration, de raffinage et d'estérification génèrent en moyenne $250 \mathrm{~kg}$ éq $\mathrm{CO}_{2} / \mathrm{t}$;

- devant la diversité des résultats des sites de lipochimie, il n'a pas été possible de dégager une tendance: un site génère seulement $20 \mathrm{~kg}$ éq $\mathrm{CO}_{2} / \mathrm{t}$, mais il s'agit d'un bilan « partiel » qui ne tient pas compte de l'ensemble du fret et des emballages, un autre génère environ $500 \mathrm{~kg}$ éq $\mathrm{CO}_{2} / \mathrm{t}$ et un troisième environ $1000 \mathrm{~kg}$ éq $\mathrm{CO}_{2} / \mathrm{t}$.

Une approche plus précise peut être effectuée à partir de la répartition, pour chaque site étudié, des émissions en fonction de ses différentes activités :

- la collecte des sous-produits d'origine animale génère des émissions comprises entre 10 et $50 \mathrm{~kg}$ éq $\mathrm{CO}_{2} / \mathrm{t}$ de matière collectée, suivant le périmètre géographique de la collecte ;

- le procédé de transformation des sousproduits d'origine animale génère des émissions comprises entre 70 et $85 \mathrm{~kg}$ éq $\mathrm{CO}_{2} / \mathrm{t}$ de matière traitée ; pour un des sites étudiés, ce procédé génère $200 \mathrm{~kg}$ éq $\mathrm{CO}_{2} / \mathrm{t}$ de matières traitées, en raison de l'usage important du fuel lourd;
- le procédé de trituration des graines oléagineuses génère de 50 à $130 \mathrm{~kg}$ éq $\mathrm{CO}_{2} / \mathrm{t}$ de graines triturées; l'écart important entre les deux valeurs provient des différences de capacité de traitement des sites considérés (il existe un facteur 4 entre la capacité de production du plus petit site et celle du plus grand) et de l'incertitude relative à l'attribution des émissions à un procédé spécifique quand plusieurs activités sont exercées sur le même site ;

- le procédé de raffinage des huiles brutes génère de 30 à $120 \mathrm{~kg}$ éq CO $/ \mathrm{t}$ d'huile brute ; l'écart important entre les deux valeurs provient des différences de capacité de traitement des sites considérés (il existe un facteur 14 entre la capacité de raffinage du plus petit site et celle du plus grand!), de la forte incertitude dans I'attribution des émissions à un procédé spécifique quand d'autres activités que le raffinage sont exercées sur le même site, mais aussi du fait que nous n'avons pas distingué le semiraffinage du raffinage ;

- l'activité de conditionnement des huiles végétales génère environ $200 \mathrm{~kg}$ éq $\mathrm{CO}_{2} / \mathrm{t}$ d'huile conditionnée (cette valeur ne concerne qu'un seul site) ;

- l'activité de production d'esters méthyliques d'huiles végétales génère entre 200 et $300 \mathrm{~kg}$ éq $\mathrm{CO}_{2} / \mathrm{t}$ d'huile (l'écart entre les deux valeurs est liée aux différences de capacité des sites, mais aussi aux types de catalyse mis en œuvre).

Ces valeurs sont données à titre indicatif et nécessitent d'être confirmées par une étude portant sur un plus grand nombre de sites industriels.

La vulnérabilité des entreprises est très majoritairement liée aux filières amont. Les émissions issues des matières premières représentent, pour tous les cas étudiés, au minimum $60 \%$ des émissions globales, avec des variations selon les sous-secteurs : entre 90 et $99 \%$ pour le traitement des sous-produits d'origine animale, de 62 à $66 \%$ dans le secteur de la lipochimie, environ $80 \%$ pour la trituration des graines oléagineuses, entre 60 et $70 \%$ pour le raffinage des huiles végétales, près de $70 \%$ pour les sites de trituration et d'estérification.

Si l'on ne tient pas compte de l'impact de la matière première, les principales émissions sont liées au fret routier, qui peut représenter plus de $80 \%$ des émissions du site et, dans la majorité des cas étudiés, plus du tiers des émissions du site. Aux consommations d'énergies sur le site, principalement le gaz, qui dans la moitié des cas étudiés représentent au moins $40 \%$ des émissions. Aux emballages qui, dans certains cas spécifiques (lipochimie, conditionnement des huiles sur les sites de raffinage), peuvent avoir un impact important (de 20 à $50 \%$ des émissions) en raison des forts facteurs d'émission associés aux matières plastiques ou métalliques.

Des actions de réduction des émissions de GES ont été proposées aux exploitants des sites industriels qui ont souhaité s'engager dans la démarche de comptabilisation des émissions liées à leur activité.

Les axes possibles de réduction des émissions de GES se situent, selon les sites, au niveau du choix de biocombustibles, du traitement des sous-produits par méthanisation avec récupération du biogaz produit pour les besoins énergétiques du site, de l'orientation du fret vers les transports ferroviaire, maritime et fluvial. Dans le secteur de la lipochimie, les émissions de GES liées aux matières premières peuvent être réduites par une réduction des quantités de matières $d^{\prime}$ 'origine pétrolière au profit de matières d'origine végétale : développement de biolubrifiants, formulation de nouveaux produits sur base végétale.

Les points de vulnérabilité identifiés amènent à nous interroger de manière plus globale sur les garanties de pérennité de la filière :

- Pour la production des matières premières, les filières agricoles sont triplement menacées: augmentation du coût des matières d'origine fossile, futures contraintes réglementaires liées aux émissions de GES, perturbation des rendements en raison des changements climatiques : I'hypothèse d'un réchauffement supérieur à $3{ }^{\circ} \mathrm{C}$ provoque, quel que soit le scénario, des chutes sérieuses des rendements des productions agricoles [20] ; quelles mesures d'adaptation sont prévues par ces filières?

- Quelles alternatives pouvons-nous trouver au fret routier? L'épuisement des ressources pétrolières et la réglementation des émissions de GES liées au transport aura pour conséquence une augmentation du coût des transports routiers et leur limitation; comment, dans ces circonstances, garantir un approvisionnement en matières premières et consommables, et l'expédition de la production vers les clients?

- Comment garantir la production de vapeur tout en réduisant la consommation de produits d'origine pétrolière?

- Comment continuer à conditionner les produits finis tout en réduisant les consommations de matières plastiques et de métaux non recyclés?

La quantification des émissions de GES de son entreprise permet donc d'identifier ses points de vulnérabilité afin de mettre en œuvre les mesures d'adaptation aux conséquences du changement climatique.

Remerciements. Ces opérations groupées ont pu être réalisées grâce au soutien financier de I'ADEME (Agence de l'Environnement et de la Maitrise de l'Energie) et de I'ONIGC (Office Natio- 
nal Interprofessionnel des Grandes Cultures). Nous remercions Sylvie PADILLA, Guy-Franck MERLOT, Pierre GALIO (ADEME) et Monique MEIZELS (ONIGC). Nous remercions également les coordinateurs des opérations: Franck JOLIBERT (UNGDA) et Ginés MARTINEZ (Réseau CTI).

\section{RÉFÉRENCES}

1. Groupe d'experts intergouvernemental su l'évolution du climat (2001). XVIII' session du GIEC. Wembley, Royaume-Uni, 24-29 septembre 2001.

2. IPPC. Climate Change 2001 : The Scientific Basis. Contribution of Working Group I to the Third Assessment Report of the Intergovernmental Panel on Climate Change. Cambridge, United Kingdom and New York, NY, USA : Cambridge University Press, 2001

3. SOLOMONS, QIND, MANNINGM, et al. Summary for Policymakers. In : Climate Change 2007 : The Physical Science Basis. Contribution of Working Group I to the Fourth Assessment Report of the Intergovernmental Panel on Climate Change. Cambridge, United Kingdom and New York : Cambridge University Press, 2007.

4. CITEPA. Rapport d'inventaire national: inventaire des émissions de gaz à effet de serre en France au titre de la convention cadre des Nations Unies sur les changements climatiques. Synthèse CCNUCC/CRF. 2006

5. Directive du Parlement européen et du Conseil $n^{\circ}$ 2003/87/CE du 13 octobre 2003 établissant un système d'échange de quotas d'émission de gaz à effet de serre dans la Communauté et modifiant la directive 96/61/CE du Conseil. 10 Commun Eur 2003 ; L : 275.
6. Décret $n^{\circ}$ 2004-832 du 19 août 2004 pris pour I'application des articles L. 229-5 à L. 229-19 du code de l'environnement et relatif au système d'échange de quotas d'émission de gaz à effet de serre. J0, 21 août 2004.

7. Décret n²007-979 du 15 mai 2007 approuvant le plan national d'affectation des quotas d'émission de gaz à effet de serre établi pour la période 2008-2012. JO, 16 mai 2007.

8. Proposition de directive du Parlement européen et du Conseil COM (2006) 818 final du 20 décembre 2006 modifiant la directive 2003/87/CE afin d'intégrer les activités aériennes dans le système communautaire d'échange de quotas d'émission de gaz à effet de serre. Non publiée au Journal Officiel de I'Union Européenne. 2006.

9. DE BOISSIEU C. Rapport du groupe de travail "Division par quatre des émissions de gaz à effet de serre de la France à l'horizon 2050 ». 2006.

10. AFNOR. Gaz à effet de serre - Partie 1 : spécifications et lignes directrices, au niveau des organismes, pour la quantification et la déclaration des émissions et des suppressions des gaz à effet de serre. NF ISO 14064-1 juin 2006. Indice de classement : X30-364-1.

11. AFNOR. Gaz à effet de serre - Partie 2 : spécifications et lignes directrices, au niveau des projets, pour la quantification, la surveillance et la déclaration des réductions d'émissions ou d'accroissements de suppressions des gaz à effet de serre. NF ISO 14064-2 Juin 2006. Indice de classement : X30-364-2.

12. AFNOR. Gaz à effet de serre - Partie $3:$ spécifications et lignes directrices pour la validation et la vérification des déclarations des gaz à effet de serre. NF ISO 14064-3 Juin 2006. Indice de classement : X30-364-3.
13. BLANCHARD O, PARODI S. Bilan gaz à effet de serre: méthodologie et application au cas de I'UPMF. Papier de travail pour le Projet d'Université Citoyenne. 2004.

14. ADEME/DIREM. Bilans énergétiques et gaz à effet de serre des filières de production de biocarburants. 2002.

15. MARTINEZ-SOSA D, RAFRAFI Y, TORRIJOS M, BERNET N, BUITRON G, BOSQUE F, DELGENES JP. Anaerobic digestion of gelatinous water at laboratory and pilot scale and nitrogen inhibition. (soumis à publication).

16. BOSQUE F. Valorisation des déchets gras de I'industrie agro-alimentaire: quels premiers enseignements? Quelles perspectives? In : Journées EFE : Nouveaux enjeux et débouchés énergétiques pour la biomasse, $4 \& 5$ avril 2006, Paris.

17. UNGDA. Rapport final "GESSICA »: Gaz à Effet de Serre : Système Intégré pour la Comptabilisation dans les industries Alimentaires. 2007.

18. Ministère des transports, de l'équipement, du tourisme et de la mer. Transports routiers de marchandises: charte d'engagements volontaires de réduction des émissions de $\mathrm{CO}_{2}$ dans le transport routier de marchandises. 2007.

19. ALFOS C, GALLIERE B. Les agro-molécules multifonctionnelles: valeur ajoutée des esters méthyliques de tournesol oléique, des esters de carbonate de glycérol et d'acides gras. Workshop OLEOMA, 27 mars 2007, Toulouse.

20. SEGUIN B. Changement climatique: de la science à l'action. Les conséquences pour l'agriculture. Responsabilité \& Environnement $2007 ; 47: 35-42$ 